\title{
Gene-set enrichment analysis of differentially expressed genes in the transcriptome of the human corneal endothelium in association with age effect
}

Sarbojoy Saha ${ }^{1}$, Shampa Barmon ${ }^{2}$

1. Department of Biochemistry and Microbiology, North south University, Dhaka, Bangladesh.

2. Department of Environmental Science and Management, North south University, Dhaka, Bangladesh.

*Corresponding author: Sarbojoy Saha, Email: sarbojoy.saha@northsouth.edu

\begin{abstract}
The eyes are the sense organs through we view the world around us, and the cornea is the transparent layer which covers the outer, visible part of the eye. It is known that the gene expression of corneal endothelium depends on age. And the expressed genes of the endothelium of cornea for pediatric samples is different than that of the adult samples. The purpose or objective of this study was to characterize human corneal endothelial cell (HCEnC) gene expression and differential gene expression and to detect the expressed genes mapped to chromosomal loci associated with some corneal endothelial dystrophies. Both upregulated and downregulated genes were analyzed. For this purpose, total RNA was isolated from ex vivo corneal endothelium taken from six pediatric and five adult corneas. The complementary DNA was hybridized to the Affymetrix GeneChip 1.1 ST array. The data analysis was performed using the Enrichr software for both upregulated and downregulated genes. These are described in more detail in the results and discussion section. This study uses bioinformatics tools to identify and analyse gene sets present in the transcriptome of the corneal endothelium, and tries to find out and observe the relation of the aging effect on the corneal endothelium gene expression. The human subjects had participated voluntarily and informed consent was obtained from all before carrying out any testing procedure. Proper guidelines from the hospital ethical committee were also followed and no harmful chemicals were used on the participants. This study simply aims to raise some awareness of the given topic among the local people so that they are better able to take informed choices about their health in the near future, and also so that they seek medical help when necessary and have no inhibitions in doing so.
\end{abstract}

Keywords: corneal, pediatric, adult, corneal endothelial dystrophies, total RNA, gene expression, gene sets, transcriptome 


\section{Introduction}

Aging affects all the different tissues and organs of the body in general and the individual is affected by the several effects of aging. The cornea of the eyes is also affected by the process of aging and changes occur in the corneal epithelium. Although limited information exists on this topic to attract more than speculations and guesswork, aging is mostly associated with a reduced ability to resist physiological stresses. Changes which occur in the ocular surface renders the cornea more susceptible to various infections. There is a rise in the permeability of the epithelium (Nzekwe and Maurice 1994) which may represent a breakdown of epithelial barrier function or other factors. The changes in the distribution of integrin protein subunits can also contribute to this decrease. The alpha- 6 and beta- 4 tend to become more discontinuous with age. But, the number and distribution of these subunits seems to remain unchanged with age. Corneal cells have a decreased ability to upregulate adhesion molecules and a weakened phagocytic activity of certain white blood cells called polymorhonucleocytes(Hazlett et al. 1990) in response to aging, and this can impair the ability to resist bacterial infections.

The negative effects of aging are not only limited to the retina and the lens of the eye, (Hazlett et al. 1990)but they also affect the cornea and the ocular surface. The corneal endothelium cells are differentiated terminally and they have the role of maintaining the integrity of the corneal endothelium. A flavonoid, eriodictyol, found in citrus fruits offers long-term protection of retinal cells(RPE) against oxidative damage (Johnson, Maher, and Hanneken 2009). The aging process involves some loss of endothelial cells over an individual's lifetime, but an increased loss of endothelial cells is seen in the condition of Fuch's endothelial cell dystrophy(FECD), often associated with other complications such as edema of the cornea (Gipson 2013). It has been observed that Fuch's dystrophy is associated with mutations in several genes, but existing research has shown that they have a common way or link as they decrease the ability of endothelial cells to deal with oxidative stress(Joyce, Harris, and Zhu 2011) and this mostly leads to cell death at a faster rate than normal (Joyce, Zhu, and Harris 2009). Therefore, oxidative stress is a major cause of this particular dystrophy called Fuch's dystrophy(Jurkunas et al. 2010). It has been seen in some transgenic mice of Tet-Mev 1 strains that mitochondrial superoxide anion overproduction accelerates these kind of (Onouchi et al. 2012)age-related corneal dysfunctions. Some forms of targeted pharmacotherapy to enhance the antioxidant capacity and a reduction in ultraviolet light exposure to decrease the UV-induced oxidative stress are being investigated as therapeutic approaches to combat this problem in the recent times.

In another study conducted with volunteers it was found after several stages of testing that axial cell counts are reproducible in the same cornea after a suitable time interval, and axial and peripheral cell counts are quite similar, and also the axial cell counts of pairs of eyes are similar(Sturrock, Sherrard, and Rice 1978). It was also found that there was a gradual decrease in the cell number with age. Endothelium actually originates from the basal crest and it is part of the elaboration of the basement membrane which is banded (Waring et al. 1982). In the human cornea, a special case is seen as the fall in the central endothelial cell density indicates that a 
large decrease or loss of corneal cells occur early in postnatal development (Association for Research in Vision and Ophthalmology. et al. 1977). No significant differences in cell density were found between the right or left eyes or between male or female subjects of similar subjects (Laule et al. 1978). Present data indicates that corneal cell density in a normal eye remains unchanged from the ages of five to twenty (Hiles, Biglan, and Fetherolf 1979). Therefore, changes in the corneal endothelium, can be thought of or described as a function of age (Laing et al. 1976).

\section{Materials and methods}

\section{Ethical statement}

Informed consent was obtained before examining all the patients with a noncontact specular microscope. All the patients had participated voluntarily, and after being informed about the testing procedure in detail. The informed consent of the author and co-author were also obtained before carrying out any procedures, and after they had assumed their full responsibilities in conducting the study. No harmful chemicals or drugs were used on the patients. This study only aims to build awareness among local populations about the aging effect on the corneal endothelium, and the endothelial cell density (ECD, as the eyes are one of the most valuable sense organs. This study was conducted in accordance to the hospital approval committee (Center of Eye diseases in Vilnius University hospital Santariskiu Clinic). No harm was done to any of the patients while examining them. The author reports no conflicts of interest in this work.

\section{Identification of upregulated genes from GSE58315}

From the NCBI website GEO datasets were searched using the term 'differential gene expression' and the reference series GSE58315 was analysed with GEO2R.

For GEO2R analysis, two groups were defined which were adult and pediatric. The adult age group had five samples and the pediatric one had six samples. Using the GEO query (Davis and Meltzer 2007) and limma R (G. Smyth, Thorne, and Wettenhall 2004), GEO2R analysis was performed (G. K. Smyth and Hall Institute 2004). Top 250 differentially expressed genes were found. $P$ values were adjusted using the false discovery rate method (Benjamini and Hochberg 1995). Log 2 transformation to the data was applied. R script used to perform the calculation was obtained from the R script tab.

Enrichment analysis

Enrichment analysis of the upregulated genes, was carried out using

- MiRTarbase 2017 (Chou et al. 2018)

- TargetScan microRNA 2017 (Agarwal et al. 2015)

- TRANSFAC and JASPAR PWMs (Khan et al. 2018)

And enrichment analysis of the downregulated genes, was carried out using

- ARCHS4 TFs Coexp (Lachmann et al. n.d.) 
- TargetScan microRNA 2017 (Agarwal et al. 2015)

- KEGG 2016 (Kanehisa et al. 2017)

- Reactome 2016 (Fabregat et al. 2017)

\section{Results and Discussion}

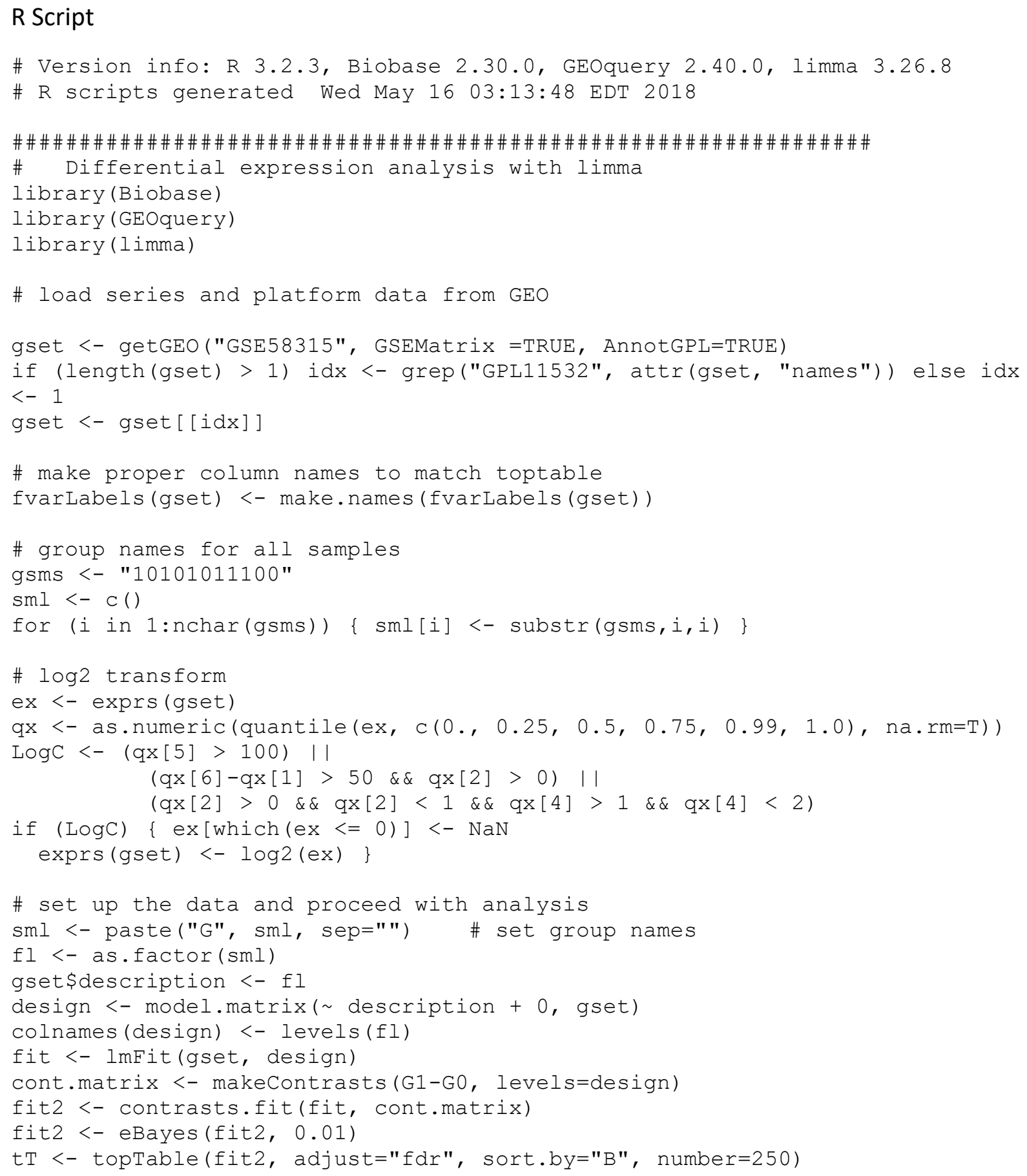






Enrichment analysis of the upregulated genes

\begin{tabular}{|c|c|c|c|c|c|}
\hline \multicolumn{6}{|c|}{ miRTarBase 2017} \\
\hline Index & Name & P-value & $\begin{array}{l}\text { Adjusted } p \text { - } \\
\text { value }\end{array}$ & Z-score & Combined score \\
\hline 1 & hsa-miR-934 & 0.004592 & 0.04362 & -2.76 & 14.88 \\
\hline 2 & $\begin{array}{l}\text { mmu-miR- } \\
3090-3 p\end{array}$ & 0.004592 & 0.04362 & -2.37 & 12.76 \\
\hline 3 & hsa-miR-3657 & 0.008175 & 0.04362 & -2.55 & 12.26 \\
\hline 4 & hsa-miR-3618 & 0.006186 & 0.04362 & -2.39 & 12.14 \\
\hline 5 & $\begin{array}{l}\text { hsa-miR- } \\
3150 a-5 p\end{array}$ & 0.005389 & 0.04362 & -2.31 & 12.08 \\
\hline
\end{tabular}




\begin{tabular}{|c|c|c|c|c|c|}
\hline \multicolumn{6}{|c|}{ TargetScan microRNA 2017} \\
\hline Index & Name & P-value & $\begin{array}{l}\text { Adjusted } p \text { - } \\
\text { value }\end{array}$ & Z-score & Combined score \\
\hline 1 & mmu-let-7a & 0.003499 & 0.0799 & -3.48 & 19.68 \\
\hline 2 & mmu-let-7b & 0.003499 & 0.0799 & -3.46 & 19.59 \\
\hline 3 & mmu-let-7c & 0.003499 & 0.0799 & -3.46 & 19.58 \\
\hline 4 & mmu-let-7d & 0.003499 & 0.0799 & -3.45 & 19.53 \\
\hline 5 & mmu-let-7e & 0.003499 & 0.0799 & -3.44 & 19.47 \\
\hline \multicolumn{6}{|c|}{ TRANSFAC and JASPAR PWMs } \\
\hline Index & Name & P-value & $\begin{array}{l}\text { Adjusted } p \text { - } \\
\text { value }\end{array}$ & Z-score & Combined score \\
\hline 1 & $\begin{array}{l}\text { NR1H3 } \\
\text { (human) }\end{array}$ & 0.001257 & 0.09427 & -1.8 & 12.05 \\
\hline 2 & $\begin{array}{l}\text { HOXA5 } \\
\text { (human) }\end{array}$ & 0.01197 & 0.2322 & -1.89 & 8.35 \\
\hline 3 & RORB (human) & 0.01497 & 0.2322 & -1.79 & 7.52 \\
\hline 4 & $\begin{array}{l}\text { NR2F1 } \\
\text { (human) }\end{array}$ & 0.01605 & 0.2322 & -1.8 & 7.46 \\
\hline 5 & $\begin{array}{l}\text { APEX1 } \\
\text { (human) }\end{array}$ & 0.02574 & 0.2322 & -1.74 & 6.37 \\
\hline
\end{tabular}

\section{Enrichment analysis for the downregulated genes}

\begin{tabular}{|c|c|c|c|c|c|}
\hline \multicolumn{6}{|c|}{ ARCHS4 TFs Coexp } \\
\hline Index & Name & P-value & $\begin{array}{l}\text { Adjuste } \\
d p- \\
\text { value }\end{array}$ & $\begin{array}{l}\text { Z- } \\
\text { scor } \\
\text { e }\end{array}$ & $\begin{array}{l}\text { Combine } \\
\text { d score }\end{array}$ \\
\hline 1 & TCF21_human_tf_ARCHS4_coexpression & 0.00131 & $\begin{array}{l}0.00893 \\
4\end{array}$ & - & 10.99 \\
\hline 2 & $\begin{array}{l}\text { SOX18_human_tf_ARCHS4_coexpressio } \\
\mathrm{n}\end{array}$ & 0.00131 & $\begin{array}{l}0.00893 \\
4\end{array}$ & $-\overline{1} \cdot 65$ & 10.95 \\
\hline 3 & $\begin{array}{l}\text { CREB3L1_human_tf_ARCHS4_coexpressi } \\
\text { on }\end{array}$ & 0.00131 & $\begin{array}{l}0.00893 \\
4\end{array}$ & - & 10.93 \\
\hline 4 & EPAS1_human_tf_ARCHS4_coexpression & 0.00131 & $\begin{array}{l}0.00893 \\
4\end{array}$ & - & 10.86 \\
\hline 5 & $\begin{array}{l}\text { PRRX2_human_tf_ARCHS4_coexpressio } \\
\mathrm{n}\end{array}$ & 0.00131 & $\begin{array}{l}0.00893 \\
4\end{array}$ & - & 10.78 \\
\hline \multicolumn{6}{|c|}{ TargetScan microRNA 2017} \\
\hline Index & Name & P-value & $\begin{array}{l}\text { Adjuste } \\
d p- \\
\text { value }\end{array}$ & $\begin{array}{l}\text { Z- } \\
\text { scor } \\
\text { e }\end{array}$ & $\begin{array}{l}\text { Combine } \\
\text { d score }\end{array}$ \\
\hline
\end{tabular}




\begin{tabular}{|c|c|c|c|c|c|}
\hline 1 & mmu-miR-3096b-3p & 0.05185 & 0.3438 & $\begin{array}{ll}- \\
3.46\end{array}$ & 10.24 \\
\hline 2 & mmu-miR-490-3p & 0.04832 & 0.3438 & $\begin{array}{l}- \\
3.27\end{array}$ & 9.9 \\
\hline 3 & mmu-miR-3070a & 0.03747 & 0.3438 & $\begin{array}{l}- \\
2.86\end{array}$ & 9.4 \\
\hline 4 & mmu-miR-3070b-3p & 0.03747 & 0.3438 & $\begin{array}{ll}- \\
2.86\end{array}$ & 9.38 \\
\hline 5 & mmu-miR-5108 & 0.03515 & 0.3438 & 2.73 & 9.13 \\
\hline \multicolumn{6}{|c|}{$\begin{array}{l}\text { Pathway } \\
\text { s }\end{array}$} \\
\hline \multicolumn{6}{|c|}{ KEGG 2016} \\
\hline Index & Name & P-value & $\begin{array}{l}\text { Adjuste } \\
d p- \\
\text { value }\end{array}$ & $\begin{array}{l}\text { Z- } \\
\text { scor } \\
\text { e }\end{array}$ & $\begin{array}{l}\text { Combine } \\
\text { d score }\end{array}$ \\
\hline 1 & $\begin{array}{l}\text { Glutathione metabolism Homo } \\
\text { sapiens_hsa00480 }\end{array}$ & 0.01036 & 0.04615 & $\begin{array}{l}- \\
1.89\end{array}$ & 8.65 \\
\hline 2 & $\begin{array}{l}\text { p53 signaling pathway Homo } \\
\text { sapiens_hsa04115 }\end{array}$ & 0.01373 & 0.04615 & $\begin{array}{l}- \\
1.74\end{array}$ & 7.45 \\
\hline 3 & $\begin{array}{l}\text { Pyrimidine metabolism Homo } \\
\text { sapiens_hsa00240 }\end{array}$ & 0.02084 & 0.04615 & $\begin{array}{ll}- \\
1.73\end{array}$ & 6.72 \\
\hline 4 & $\begin{array}{l}\text { Systemic lupus erythematosus Homo } \\
\text { sapiens_hsa05322 }\end{array}$ & 0.02673 & 0.04615 & $\begin{array}{ll}- \\
1.67\end{array}$ & 6.06 \\
\hline 5 & Alcoholism Homo sapiens_hsa05034 & 0.03532 & 0.04615 & $\begin{array}{ll}- \\
1.78\end{array}$ & 5.94 \\
\hline \multicolumn{6}{|c|}{ Reactome 2016} \\
\hline Index & Name & P-value & $\begin{array}{l}\text { Adjuste } \\
d p- \\
\text { value }\end{array}$ & $\begin{array}{l}\text { Z- } \\
\text { scor } \\
\text { e }\end{array}$ & $\begin{array}{l}\text { Combine } \\
\text { d score }\end{array}$ \\
\hline 1 & $\begin{array}{l}\text { Detoxification of Reactive Oxygen } \\
\text { Species Homo sapiens_R-HSA-3299685 }\end{array}$ & $\begin{array}{l}0.00658 \\
4\end{array}$ & 0.02963 & $\begin{array}{ll}- \\
2.33\end{array}$ & 11.69 \\
\hline 2 & $\begin{array}{l}\text { Synthesis and interconversion of } \\
\text { nucleotide di- and triphosphates Homo } \\
\text { sapiens_R-HSA-499943 }\end{array}$ & $\begin{array}{l}0.00499 \\
1\end{array}$ & 0.02963 & -2.1 & 11.13 \\
\hline 3 & $\begin{array}{l}\text { TP53 Regulates Metabolic Genes Homo } \\
\text { sapiens_R-HSA-5628897 }\end{array}$ & 0.0167 & 0.0389 & -2 & 8.17 \\
\hline 4 & $\begin{array}{l}\text { Metabolism of nucleotides Homo } \\
\text { sapiens_R-HSA-15869 }\end{array}$ & 0.01729 & 0.0389 & $\begin{array}{l}- \\
2.01\end{array}$ & 8.17 \\
\hline 5 & $\begin{array}{l}\text { Cellular responses to stress Homo } \\
\text { sapiens_R-HSA-2262752 }\end{array}$ & 0.07141 & 0.1071 & - & 6.14 \\
\hline
\end{tabular}




\section{For the upregulated genes:}

The hsa-miR-934 was found to be the most significant miRNA from the miRTarBase 2017. From the TargetScan micro RNA 2017 database, mmu-let-7a was the most significant gene. NR1H3(human) was found to be the most significant transcription factor from the TRANSFAC and JASPAR PWMs transcription factor binding profile.

\section{For the downregulated genes:}

TCF21_human_tf_ARCHS4_coexpression was discovered to be the most significant transcription factor from the ARCHS4 TFs database. From the TargetScan microRNA database, mmu-miR3096b-3p was the most significant microRNA. Glutathione metabolism Homo sapiens_hsa00480 was found to be the most significant pathway from the KEGG 2016 pathway. And finally from the Reactome 2016 pathway the most significant pathway was seen to be Detoxification of Reactive Oxygen Species Homo sapiens_R-HSA-3299685.

It can be observed that several databases from the Enrichr website gave significant data or values of genes, transcription factors and microRNAs and pathways. Three databases gave significant data for the upregulated genes, and four gave significant values for the downregulated genes. So there was availability of sufficient significant transcription factors, microRNAs and pathways. Gene set enrichment analysis using available software like Enrichr Mayaan Laboratory enables us to understand the transcription factors, pathways, ontologies, proteins, diseases and drugs and others. The current study has tried to do just that for the differentially expressed genes of the transcriptome of corneal endothelium. Out of the different databases shown here in the table the TargetScan microRNA is common for both the upregulated and the downregulated genes. So, it can be understood that microRNAs are an important component in this gene set analysis of differentially expressed genes for the cornea endothelium. also the ARCHS4 TFs Coexp or transcription factors Coexpression have significant values, along with the KEGG and Reactome pathways. In the KEGG pathway, e.g. Glutathione metabolism Homo sapiens_hsa00480 the most significant pathway indicating the great importance of the glutathione metabolism pathway in humans and Systemic lupus erythematosus Homo sapiens_hsa05322 is another significant pathway here. Nowadays. Systemic lupus erythematosus is quite prevalent in the world, even in the developed world.

\section{Conclusion}

The eye is a very valuable sense organ of the human body. The eye has several different parts with different functions, cornea being one of those parts. Basically, cornea is the transparent layer in the front outer layer of the eyes. It is a critical area of the eye as light passes through it to the inner parts and this then enables us to see. The corneal endothelium transcriptome has differentially expressed genes, both upregulated and downregulated genes. The gene set enrichment analysis carried out in this study has tried to identify the differentially expressed genes and the different transcription factors and pathways involved using the Enrichr website. 
Hopefully, such knowledge will help to understand the effects of aging on the corneal endothelium, and this will be able to raise some awareness of the effects of the aging process on the cornea among the general public so that they take good care of their eyes and take regular medical check -ups. This study has aimed to build this awareness among the local populations and hopefully this will help them to take informed decisions in the future. 


\section{REFERENCES}

Agarwal, Vikram, George W Bell, Jin-Wu Nam, and David P Bartel. 2015. "Predicting Effective MicroRNA Target Sites in Mammalian MRNAs." ELife 4 (August). elife Sciences Publications, Ltd. https://doi.org/10.7554/eLife.05005.

Association for Research in Vision and Ophthalmology., C F, R M Glassman, D K MacCallum, J H Lillie, R F Meyer, B J Robinson, and N M Rich. 1977. Investigative Ophthalmology \&amp; Visual Science. Investigative Ophthalmology \& Visual Science. Vol. 27. C.V. Mosby Co. http://iovs.arvojournals.org/article.aspx?articleid=2159864.

Benjamini, Yoav, and Yosef Hochberg. 1995. "Controlling the False Discovery Rate: A Practical and Powerful Approach to Multiple Testing." Source Journal of the Royal Statistical Society. Series B (Methodological) 57 (1): 289-300. http://www.jstor.org/stable/2346101.

Chou, Chih-Hung, Sirjana Shrestha, Chi-Dung Yang, Nai-Wen Chang, Yu-Ling Lin, Kuang-Wen Liao, Wei-Chi Huang, et al. 2018. "MiRTarBase Update 2018: A Resource for Experimentally Validated MicroRNA-Target Interactions." Nucleic Acids Research 46 (D1): D296-302. https://doi.org/10.1093/nar/gkx1067.

Davis, S., and P. S. Meltzer. 2007. "GEOquery: A Bridge between the Gene Expression Omnibus (GEO) and BioConductor." Bioinformatics 23 (14): 1846-47. https://doi.org/10.1093/bioinformatics/btm254.

Fabregat, Antonio, Konstantinos Sidiropoulos, Guilherme Viteri, Oscar Forner, Pablo MarinGarcia, Vicente Arnau, Peter D’Eustachio, Lincoln Stein, and Henning Hermjakob. 2017. "Reactome Pathway Analysis: A High-Performance in-Memory Approach." BMC Bioinformatics 18 (1). BioMed Central: 142. https://doi.org/10.1186/s12859-017-1559-2.

Gipson, llene K. 2013. "Age-Related Changes and Diseases of the Ocular Surface and Cornea." Investigative Opthalmology \& Visual Science $54 \quad$ (14): $\quad$ ORSF48. https://doi.org/10.1167/iovs.13-12840.

Hazlett, L D, F B Kreindler, R S Berk, and R Barrett. 1990. “Aging Alters the Phagocytic Capability of Inflammatory Cells Induced into Cornea." Current Eye Research 9 (2): 129-38. http://www.ncbi.nlm.nih.gov/pubmed/2335110.

Hiles, David A., Albert W. Biglan, and Edward C. Fetherolf. 1979. "Central Corneal Endothelial Cell Counts in Children." American Intra-Ocular Implant Society Journal 5 (4). Elsevier: 292-300. https://doi.org/10.1016/S0146-2776(79)80078-6.

Johnson, Jennifer, Pamela Maher, and Anne Hanneken. 2009. "The Flavonoid, Eriodictyol, Induces Long-Term Protection in ARPE-19 Cells through Its Effects on Nrf2 Activation and Phase 2 Gene Expression." Investigative Opthalmology \& Visual Science 50 (5): 2398. https://doi.org/10.1167/iovs.08-2088.

Joyce, Nancy C., Deshea L. Harris, and Cheng C. Zhu. 2011. "Age-Related Gene Response of Human Corneal Endothelium to Oxidative Stress and DNA Damage." Investigative 
Opthalmology \& Visual Science 52 (3): 1641. https://doi.org/10.1167/iovs.10-6492.

Joyce, Nancy C., Cheng C. Zhu, and Deshea L. Harris. 2009. "Relationship among Oxidative Stress, DNA Damage, and Proliferative Capacity in Human Corneal Endothelium." Investigative Opthalmology \& Visual Science 50 (5): 2116. https://doi.org/10.1167/iovs.08-3007.

Jurkunas, Ula V., Maya S. Bitar, Toshinari Funaki, and Behrooz Azizi. 2010. "Evidence of Oxidative Stress in the Pathogenesis of Fuchs Endothelial Corneal Dystrophy." The American Journal of Pathology 177 (5): 2278-89. https://doi.org/10.2353/ajpath.2010.100279.

Kanehisa, Minoru, Miho Furumichi, Mao Tanabe, Yoko Sato, and Kanae Morishima. 2017. "KEGG: New Perspectives on Genomes, Pathways, Diseases and Drugs." Nucleic Acids Research 45 (D1). Oxford University Press: D353-61. https://doi.org/10.1093/nar/gkw1092.

Khan, Aziz, Oriol Fornes, Arnaud Stigliani, Marius Gheorghe, Jaime A Castro-Mondragon, Robin van der Lee, Adrien Bessy, et al. 2018. "JASPAR 2018: Update of the Open-Access Database of Transcription Factor Binding Profiles and Its Web Framework." Nucleic Acids Research 46 (D1). Oxford University Press: D260-66. https://doi.org/10.1093/nar/gkx1126.

Lachmann, Alexander, Denis Torre, Alexandra B Keenan, Kathleen M Jagodnik, Hyojin J Lee, Lily Wang, Moshe C Silverstein, and Avi Ma 'ayan. n.d. "Massive Mining of Publicly Available RNA-Seq Data from Human and Mouse." Accessed May 16, 2018. https://doi.org/10.1101/189092.

Laing, R A, M M Sanstrom, A R Berrospi, and H M Leibowitz. 1976. "Changes in the Corneal Endothelium as a Function of Age." Experimental Eye Research 22 (6): 587-94. http://www.ncbi.nlm.nih.gov/pubmed/776638.

Laule, A, M K Cable, C E Hoffman, and C Hanna. 1978. "Endothelial Cell Population Changes of Human Cornea during Life." Archives of Ophthalmology (Chicago, III. : 1960) 96 (11): 203135. http://www.ncbi.nlm.nih.gov/pubmed/718491.

Nzekwe, E U, and D M Maurice. 1994. "The Effect of Age on the Penetration of Fluorescein into the Human Eye." Journal of Ocular Pharmacology 10 (3): 521-23. http://www.ncbi.nlm.nih.gov/pubmed/7836860.

Onouchi, Hiromi, Takamasa Ishii, Masaki Miyazawa, Yuichi Uchino, Kayo Yasuda, Phil S. Hartman, Kenji Kawai, Kazuo Tsubota, and Naoaki Ishii. 2012. "Mitochondrial Superoxide Anion Overproduction in Tet - Mev-1 Transgenic Mice Accelerates Age-Dependent Corneal Cell Dysfunctions." Investigative Opthalmology \& Visual Science 53 (9): 5780. https://doi.org/10.1167/iovs.12-9573.

Smyth, Gordon K, and Eliza Hall Institute. 2004. "Statistical Applications in Genetics and Molecular Biology Linear Models and Empirical Bayes Methods for Assessing Differential Expression in Microarray Experiments." Statistical Applications in Genetics and Molecular Biology 3 (1). https://doi.org/10.2202/1544-6115.1027.

Smyth, Gordon, Natalie Thorne, and James Wettenhall. 2004. "Limma: Linear Models for Microarray

Data

User's

Guide." 
ftp://ftp.esat.kuleuven.be/pub/sista/marchal/updated_courses_masterfiles_2007/5_Micr oarray_Preprocessing/Datasets/Bioconductor/usersguide.pdf.

Sturrock, G D, E S Sherrard, and N S Rice. 1978. "Specular Microscopy of the Corneal Endothelium." The British Journal of Ophthalmology 62 (12): 809-14. http://www.ncbi.nlm.nih.gov/pubmed/737161.

Waring, G O, W M Bourne, H F Edelhauser, and K R Kenyon. 1982. "The Corneal Endothelium. Normal and Pathologic Structure and Function." Ophthalmology 89 (6): 531-90. http://www.ncbi.nlm.nih.gov/pubmed/7122038. 